\title{
EDITORIAL
}

\section{Venous Thromboembolism Prophylaxis in the United States: Still Room for Improvement}

\author{
Diane Sliwka, MD and Margaret C. Fang, MD, MPH \\ Division of Hospital Medicine, The University of California San Francisco, San Francisco, CA, USA.
}

$J$ Gen Intern Med 25(6):484-6

DOI: $10.1007 / \mathrm{s} 11606-010-1350-9$

(c) The Author(s) 2010. This article is published with open access at Springerlink.com

$\mathrm{V}$ enous thromboembolism (VTE) is one of the most common, life-threatening cardiovascular conditions in the United States, causing approximately 200,000 deaths each year. ${ }^{1,2}$ Hospitalized patients are particularly vulnerable to VTE, with approximately $42.5 \%$ of all hospitalized medical patients considered to be at increased VTE risk. ${ }^{3}$ As a result, multiple guidelines, including the prominent American College of Chest Physicians Guidelines on Antithrombotic and Thrombolytic Therapy, support the administration of measures to prevent VTE in appropriate patients. ${ }^{4}$ Although pharmacologic and, to a lesser degree, mechanical forms of prophylaxis, have been shown to significantly reduce the risk of $\mathrm{VTE}^{5}$, several studies have shown that many hospitalized patients do not receive VTE prophylaxis. ${ }^{6-8}$ In this issue of the Journal of General Internal Medicine, Rothberg et al. present a large analysis of VTE prophylaxis rates among medical patients admitted to 376 acute-care facilities in the United States, finding that a striking $64 \%$ of patients with at least a moderate risk of VTE failed to receive VTE prophylaxis by the second day of their hospitalization. ${ }^{9}$

Using an administrative database of greater than 350,000 medical inpatients with primary diagnoses indicating a moderate to high-risk of VTE, this study revealed not only overall low rates of VTE prophylaxis, but also considerable variability in utilization rates across hospitals and providers. Despite some differences in patient selection and definition of VTE prophylaxis, the low administration rates are consistent with other similar multi-institutional studies. ${ }^{6,10,11}$ A unique finding of the study was that, although prophylaxis rates varied among individual physicians, physician practice seemed to cluster around institutional means. Patients with similar VTE risk profiles had widely divergent likelihoods of receiving VTE prophylaxis depending on the admitting hospital, with variability observed by hospital size, type, and region. This variation in clinical practice among hospitals suggests that strategies aimed at improving prophylaxis rates should be directed at the institutional level.

There are several limitations to this observational study using administrative data. One is the lack of information on clinical decision-making with regard to VTE prophylaxis, such

Published online February 14, 2010 as contraindications to pharmacologic VTE prophylaxis or patient preference. For example, a prior study found that contraindications excluded $10 \%$ of medical patients from receiving pharmacologic VTE prophylaxis. ${ }^{6}$ It was possible that some services may not have been consistently or uniformly captured, as they were dependent on accurate billing and utilization codes. Finally, using ICD-9 coded clinical diagnoses to classify patients into varying levels of VTE risk may be subject to misclassification and cannot completely capture the unique circumstances of individual patients, such as comfort care or highly ambulatory individuals who were thought to be at low VTE risk.

Nevertheless, even allowing that some patients in this analysis may not have required or desired VTE prophylaxis, the low administration rates in the study still suggest that significant numbers of patients fail to receive appropriate VTE prophylaxis. That variation in prophylaxis administration seemed to be more dependent on region and hospital practice as opposed to individual patient factors highlights the need for continued study in system-wide and institutionally-directed interventions. The direction of further research should now focus less on describing the scope of the problem and more on how to best address barriers to quality improvement and most effectively implement solutions.

\section{STRATEGIES TO IMPROVE VTE PROPHYLAXIS}

Expert panels, national quality organizations, and regulatory bodies have aligned to address VTE prevention. The Joint Commission, the National Quality Forum, the Centers for Medicare \& Medicaid Services, and the Agency for Healthcare Research and Quality (AHRQ) have developed measure-based initiatives such as quality indicators, public reporting, and incentive and disincentive programs targeting VTE prophylaxis rates. These have provided the impetus for national collaboratives such as the Society of Hospital Medicine (SHM) VTE Prevention Collaborative ${ }^{12}$ and the incorporation of VTE prophylaxis in the Surgical Care Improvement Project (SCIP). The Surgeon General issued a "Call to Action to Prevent Deep Venous Thrombosis and Pulmonary Embolism" in 2008, further emphasizing VTE prevention as a major public health concern. ${ }^{13}$ Despite efforts from leading regulatory and quality improvement agencies to promote awareness and motivation for VTE prophylaxis, as well as clear evidence that the impact of VTE prevention efforts can be large, what seem to be straightforward interventions are frequently challenging to implement in actual practice. ${ }^{14}$

The simplest but probably least effective strategy is attempting to change practice through passive methods such as the 
development and publication of guidelines. Although guidelines are vital in summarizing best practice recommendations and expert opinion, even well-known guidelines, such as those recommending VTE prophylaxis, face multiple barriers in terms of assimilation into clinical practice. ${ }^{9,15-17}$ The dissemination of locally specific recommendations can be more effective, particularly as part of a multipronged strategy. ${ }^{18}$

More active strategies, such as targeted education about VTE prophylaxis, ${ }^{19}$ have shown effectiveness in improving VTE prophylaxis but are limited in reach and retention over time. Relying on individual clinicians to remember and apply information correctly at each clinical encounter is inefficient and frequently ineffective. An important area for further research should be directed in how to best implement 'micro' level (i.e., at the point of care) interventions to assist providers at the time of the clinical encounter so as to encourage specific behaviors. Examples of micro-level interventions that have been shown to improve VTE prophylaxis rates include automated reminder systems such as computer alerts ${ }^{20}$ and protocols embedded in admission and transfer order sets. ${ }^{20}$ Computer alerts have been shown to improve VTE prophylaxis ordering rates ${ }^{23,24}$ and were associated with a $41 \%$ reduction in the 90-day incidence of VTE in one study. ${ }^{20}$ Successful strategies acknowledge that clinical care is complex and fastpaced, and attempt to integrate interventions into usual clinical workflow. Interventions that embed VTE prophylaxis into standardized, pre-existing order sets are more effective than asking physicians to complete separate, free-standing VTE-specific order sets. ${ }^{12}$ Simple interventions such as checklists may also be effective, having been shown to dramatically improve clinical outcomes in other settings such as reducing catheter-related blood stream infections ${ }^{21}$, postoperative complications, and surgery-related deaths. ${ }^{22}$

Enhancing clinical decision-support through building in reminder-systems and evidence-based recommendations into computerized order sets and electronic medical records, has great potential. ${ }^{25}$ A 2005 meta-analysis of such systems found improvements in specific processes of care $62 \%$ of the time, though only $13 \%$ showed improved patient outcomes. Barriers to effective use included poor workflow integration, misaligned relevance to patients at the point of care, and provider disagreement with recommendations. ${ }^{25-27}$ Importantly, the variability in uptake of computerized medical record systems as well as the heterogeneity in decision-support capability across specific institutions are barriers to widespread adoption of this strategy at present.

Measurement, through real time audit and feedback, is an essential component of any improvement strategy. Combining audit and feedback with other effective strategies such as reminder systems and decision support at the point of care, have been shown to improve VTE prophylaxis to near $100 \% .^{12}$ However, there are numerous challenges in being able to collect data in real time such that individual providers can respond to audit and feedback. One such intervention, the use of a 'quality dashboard' to provide summary reports of predetermined clinical measures, is an example of a specific tool that can contribute to the success of high performing institutions. ${ }^{28}$ Quality dashboards can help hospital leadership guide the quality agenda, create accountability for high-priority measures ${ }^{29}$ and provide actionable information to effect change at the point-of-care. ${ }^{30}$

Finally, catalyzing real improvement across institutions would benefit from the adoption of general frameworks of effective quality improvement strategies. One example is the VTE Prevention Collaborative supported by the Society of Hospital Medicine, the Institute for Healthcare Improvement, and AHRQ. ${ }^{12}$ This collaborative seeks to disseminate effective strategies shown to improve VTE care as well as provide guidance and mentorship to individual hospitals at the local level to help address barriers to implementation ${ }^{12}$. By pooling knowledge and best practices, providing specific toolkits for quality improvement, and tailoring solutions to local institutional culture, such initiatives help disseminate effective solutions and reduce duplication of efforts. Identifying characteristics of high-performing institutions can provide templates for other hospitals to model their efforts. Features of high-performing institutions include a culture that welcomes quality improvement and change, supports and retains individual quality improvement "champions", and institutional transparency and accountability. ${ }^{28}$

\section{CONCLUSION}

Significant numbers of hospitalized patients in the United States fail to receive appropriate VTE prophylaxis despite clear guidelines and widespread efforts to raise VTE awareness. The study by Rothberg et al. highlights the magnitude and widespread nature of the problem, as well as the wide variability in practice across different institutions ${ }^{9}$. Efforts should now focus on disseminating effective strategies to change clinical practice, both at the point of care and institutional level, and seek out high-performing institutions as models of effective practice change.

Open Access: This article is distributed under the terms of the Creative Commons Attribution Noncommercial License which permits any noncommercial use, distribution, and reproduction in any medium, provided the original author(s) and source are credited.

Corresponding Author: Diane Sliwka, MD; Division of Hospital Medicine, The University of California San Francisco, 533 Parnassus Ave., Box 0131, San Francisco, CA 94143, USA (e-mail:dsliwka@medicine.ucsf.edu).

\section{REFERENCES}

1. Geerts WH, Pineo GF, Heit JA, et al. Prevention of venous thromboembolism: The seventh ACCP conference on antithrombotic and thrombolytic therapy. Chest. 2004;126(3 Suppl):338S-400S.

2. Anderson FA Jr, Wheeler HB, Goldberg RJ, et al. A population-based perspective of the hospital incidence and case-fatality rates of deep vein thrombosis and pulmonary embolism. the worcester DVT study. Arch Intern Med. 1991;151(5):933-8.

3. Cohen AT, Tapson VF, Bergmann JF, et al. Venous thromboembolism risk and prophylaxis in the acute hospital care setting (ENDORSE study): A multinational cross-sectional study. Lancet. 2008;371 (9610):387-94.

4. Geerts WH, Bergqvist D, Pineo GF, et al. Prevention of venous thromboembolism: American college of chest physicians evidence-based clinical practice guidelines (8th edition). Chest. 2008;133(6 Suppl):381S-453S.

5. Dentali F, Douketis JD, Gianni M, Lim W, Crowther MA. Metaanalysis: Anticoagulant prophylaxis to prevent symptomatic venous thromboembolism in hospitalized medical patients. Ann Intern Med. 2007; 146(4):278-88. 
6. Cohen AT, Tapson VF, Bergmann JF, et al. Venous thromboembolism risk and prophylaxis in the acute hospital care setting (ENDORSE study): A multinational cross-sectional study. Lancet. 2008;371 (9610):387-94

7. Tapson VF, Decousus H, Pini M, et al. Venous thromboembolism prophylaxis in acutely ill hospitalized medical patients: Findings from the international medical prevention registry on venous thromboembolism. Chest. 2007;132(3):936-45.

8. Goldhaber SZ, Tapson VF, Steering Committee. A prospective registry of 5, 451 patients with ultrasound-confirmed deep vein thrombosis. Am J Cardiol. 2004;93(2):259-62.

9. Rothberg MB, Lahti M, Pekow PS, Lindenauer PK. Venous thromboembolism prophylaxis among medical patients at US hospitals. J Gen Intern Med. 2010.

10. Amin A, Stemkowski S, Lin J, Yang G. Thromboprophylaxis rates in US medical centers: Success or failure? J Thromb Haemost. 2007;5 (8):1610-6.

11. Amin AN, Stemkowski S, Lin J, Yang G. Inpatient thromboprophylaxis use in U.S. hospitals: Adherence to the seventh american college of chest physician's recommendations for at-risk medical and surgical patients. J Hosp Med. 2009;4(8):E15-21.

12. Maynard G, Stein J. Designing and implementing effective venous thromboembolism prevention protocols: Lessons from collaborative efforts. J Thromb Thrombolysis. 2010;29(2):159-66.

13. Galson SK. The surgeon general's call for action to prevent deep venous thrombosis and pulmonary embolism. 2008;2010(2 24 2010).

14. Erasmus V, Daha TJ, Brug $\mathbf{H}$, et al. Systematic review of studies on compliance with hand hygiene guidelines in hospital care. Infect Control Hosp Epidemiol. 2010;31(3):283-94.

15. Cabana MD, Rand CS, Powe NR, et al. Why don't physicians follow clinical practice guidelines? A framework for improvement. JAMA. 1999;282(15): 1458-65.

16. Cohn SL. Prophylaxis of venous thromboembolism in the US: Improving hospital performance. J Thromb Haemost. 2009;7(9):1437-45.

17. Lomas J, Anderson GM, Domnick-Pierre K, Vayda E, Enkin MW, Hannah WJ. Do practice guidelines guide practice? the effect of a consensus statement on the practice of physicians. N Engl J Med. 1989;321(19):1306-11.
18. Peterson GM, Drake CI, Jupe DM, Vial JH, Wilkinson S. Educational campaign to improve the prevention of postoperative venous thromboembolism. J Clin Pharm Ther. 1999;24(4):279-87.

19. Michota FA. Bridging the gap between evidence and practice in venous thromboembolism prophylaxis: The quality improvement process. J Gen Intern Med. 2007;22(12): 1762-70.

20. Kucher N, Koo S, Quiroz R, et al. Electronic alerts to prevent venous thromboembolism among hospitalized patients. N Engl J Med. 2005;352 (10):969-77.

21. Pronovost $\mathbf{P}$, Needham $\mathbf{D}$, Berenholtz $\mathbf{S}$, et al. An intervention to decrease catheter-related bloodstream infections in the ICU. N Engl J Med. 2006;355(26):2725-32.

22. Haynes AB, Weiser TG, Berry WR, et al. A surgical safety checklist to reduce morbidity and mortality in a global population. N Engl $\mathrm{J}$ Med. 2009;360(5):491-9.

23. Dexter PR, Perkins S, Overhage JM, Maharry K, Kohler RB, McDonald CJ. A computerized reminder system to increase the use of preventive care for hospitalized patients. N Engl J Med. 2001;345(13):965-70.

24. Durieux P, Nizard R, Ravaud P, Mounier N, Lepage E. A clinical decision support system for prevention of venous thromboembolism Effect on physician behavior. JAMA. 2000;283(21):2816-21.

25. Garg AX, Adhikari NK, McDonald H, et al. Effects of computerized clinical decision support systems on practitioner performance and patient outcomes: A systematic review. JAMA. 2005;293(10):1223-38.

26. Reisman Y. Computer-based clinical decision aids. A review of methods and assessment of systems. Med Inform (Lond). 1996;21(3):179-97.

27. Schnipper JL, Linder JA, Palchuk MB, et al. "Smart forms" in an electronic medical record: Documentation-based clinical decision support to improve disease management. J Am Med Inform Assoc. 2008;15(4):513-23.

28. Meyer APD, Silow-Carroll SMBAMSW, Kutyla TMLA, Stepnick LS, Ribowski LS. Hospital quality: Ingredients for success overview and lessons learned. The Commonwealth Fund. 2004 July.

29. Kroch EP, Vaughn TP, Koepke MJ, MHA, et al. Hospital boards and quality dashboards. Journal of Patient Safety. 2006;2(1):10-19.

30. Stein J, Chernetsky Tejedor S, Shabbir H, O'Malley E. Situational awareness improves prevalence of VTE prophylaxis on multiple nursing units. Journal of Hospital Medicine. 2008(3(S1):41) 